\title{
COTIDIANIDADES Y SOLIDARIDADES POLÍTICAS: UNA CARTOGRAFÍA DE LA EDUCACIÓN PARA LA PAZ EN COLOMBIA ${ }^{1}$ \\ EVERYDAY AND POLITICAL SOLIDARITIES: A CARTOGRAPHY OF EDUCATION FOR PEACE IN COLOMBIA
}

\author{
Natalia Sánchez-Corrales - nasanchez@unisalle.edu.co
}

\section{RESUMEN}

La coyuntura del proceso de construcción del acuerdo de paz de la última década en Colombia ha supuesto una producción copiosa de política pública, la aparición de nuevos modos de institucionalidad y gubermentalidad, así como nuevos términos en la conversación nacional sobre lo que hemos sido y nuestras posibilidades hacia el futuro. Aunque no es una formación homogénea, pues diversos actores se han alineado en posicionalidades heterogéneas con respecto a este nuevo acuerdo, lo cierto es que ha producido unas coordenadas que serán en este texto objeto de estudio en relación con una pequeña parte de este andamiaje: la educación para la paz. Esta cartografía del poder tiene la pretensión de ubicar tanto los discursos dominantes que han hecho posible la producción de conocimiento sobre la paz, como mostrar lo que activamente producen como ausencias, lo que se silencia y queda invisible. Para evitar que este ejercicio cartográfico simplemente disponga en el espacio la operación del poder y sus efectos sobre sujetos específicos, esencializándolos y condenándolos a ser sujetos sujetados por estos discursos en operación, esta cartografía se propone también mostrar que en estas ausencias y silencios producidos hay una multiplicidad de prácticas que pueden orientar alternativas concretas, contextualizadas, cotidianas y situadas, en síntesis, proyectos emancipatorios con minúscula que pueden crear condiciones para la esperanza y la solidaridad entre las luchas.

PALABRAS CLAVE: Sociología de ausencias y emergencias, Educación para la paz, Hermenéutica feminista.

\section{RESUMO}

A conjuntura do processo de construção do acordo de paz da última década na Colômbia significou uma produção abundante de políticas públicas, o surgimento de novos modos de institucionalidade e governo, bem como novos termos na conversa nacional sobre o que temos sido e nossos possibilidades para o futuro. Embora não seja uma formação homogênea, pois vários atores se alinharam em posições heterogêneas em relação a este novo acordo, a verdade é que ele produziu algumas coordenadas que estarão neste texto em estudo em relação a uma pequena parte deste andaime: a educação para a paz. Esse mapeamento do poder tem por objetivo localizar os discursos dominantes que tornaram possível a produção de conhecimento sobre a paz e mostrar o que eles produzem ativamente como ausências, o que é silenciado e invisível. Para evitar que esse exercício cartográfico simplesmente organize no espaço a operação do poder e seus efeitos sobre assuntos específicos, essencializando-os e condenando-os a serem sujeitos mantidos por esses discursos em operação, essa cartografia também visa mostrar que nessas ausências e silêncios produzidos existe um multiplicidade de práticas que podem orientar alternativas

\footnotetext{
${ }^{1}$ Este artículo contiene resultados de un proyecto de investigación adelantado entre 2014-2018 titulado "Cartografías en acción de la educación para la paz en Colombia" en la Universidad de Los Andes y con financiación del Departamento Administrativo de Ciencia, Tecnología e Innovación - Colciencias del Gobierno de Colombia.
} 
concretas, contextualizadas, cotidianas e situadas, em suma, projetos emancipatórios com minúsculas que podem criar condições de esperança e a solidariedade entre as lutas.

PALAVRAS CHAVE: Sociologia das ausências e emergências, Educação para a paz, Hermenêutica feminista.

\section{INTRODUCCIÓN}

El campo de los estudios de paz se ha consolidado como un marco de referencia para la toma de decisiones en política pública de parte de los principales actores del sistema internacional. Con la aspiración de transformar la realidad creando capacidades en los individuos para su plena participación en las instituciones políticas y económicas, la perspectiva realista de las Relaciones Internacionales se impone sobre su correlato idealista para definir los principios en operación del campo (RICHMOND, 2010). Este realismo implica suponer que conceptos como Estado, Paz y Seguridad son realidades objetivas inmediatamente disponibles para su verificación como hechos ciertos del sistema internacional, que cuentan con mecanismos e instituciones supranacionales encargadas de regular y supervisar el cumplimiento de los acuerdos alcanzados por las partes.

La homogeneidad con la que actualmente opera este discurso sobre lo que es la paz y como se crea o construye para otros no ha existido siempre. La emergencia del campo puede rastrearse a principios de la segunda mitad del siglo XX (GRASA, 2010); pero, no será sino hasta 1992 que el marco conceptual del campo producido por Galtung (1970/1996) sea empleado como parte del aparato conceptual que el sistema de Naciones Unidas utiliza para operacionalizar sus intervenciones en los países en conflicto. Esta normalización del vocabulario, metodologías y referentes en la conversación sobre la paz a propósito de un único referente que, es además promocionado por las principales agencias de financiación en todo el sur global como mecanismo de trámite de las violencias, ha sido objeto de una multiplicidad de críticas, entre las que se cuenta la revisión de la naturaleza estandarizada del modelo que se encarga de reproducir la matriz cultural occidental en contextos culturales no-occidentales a través de la ecuación que iguala paz a democracia electoral y economía de mercado (CUNHA, 2006; PUREZA \& CRAVO, 2009). Así, se constituyen los términos en los que se define un orden socio-político-económico que los países violentos del sur deben buscar, y los del norte promover. 
Es en función de estas críticas que los estudios feministas y poscoloniales han hecho a la ciencia de la paz, que a continuación me propongo hacer una cartografía del poder que está en juego en el discurso de la educación para la paz en este momento de coyuntura en Colombia. Esta cartografía del poder tiene la pretensión de ubicar tanto los discursos dominantes sobre el tema en este contexto; como mostrar lo que activamente producen como ausencias, lo que se silencia y queda invisible (SANTOS, 2006). Para evitar que este ejercicio cartográfico simplemente disponga en el espacio la operación del poder y sus efectos sobre sujetos específicos, esencializándolos y condenándolos a ser sujetos sujetados por estos discursos en operación (PRECIADO, 2008); esta cartografía se propone también mostrar que en estas ausencias y silencios producidos hay una multiplicidad de experiencias cotidianas que pueden orientar alternativas concretas (MOHANTY, RUSSO \& TORRES, 1991), contextualizadas, historizadas y situadas, en síntesis, proyectos emancipatorios con minúscula que pueden crear condiciones para la esperanza y para tejer solidaridades entre las luchas que son descartadas por los discursos más institucionales y dominantes sobre la paz.

Con esta pretensión en mente, procederé en primer lugar a explicitar el marco teórico de mis intenciones metodológicas. En segundo lugar, presento la historia de cómo se fue tejiendo este mapa a partir de los viajes, las conversaciones, los objetos y las prácticas que fui encontrando en este proceso de investigación. En un tercer momento, me propongo demostrar que hay una serie de ausencias activamente producidas en tanto ausencias por la monocultura que iguala paz y desarrollo, para finalmente, mostrar cómo en estas cotidianidades invisibles y silenciadas, emergen una serie de alternativas que nos permiten pensar en las condiciones para la esperanza, de unas paces desde quienes han sobrevivido, permanecido y resignificado la historia.

\section{MARCO TEÓRICO-METODOLÓGICO}

Los estudios feministas, poscoloniales y posliberales han elaborado hasta hoy una crítica sistemática al proyecto universalista de la civilización occidental y en particular a su concreción en la ciencia moderna (CASTRO-GÓMEZ, 2005; CHAKRABARTI, 2008; ESCOBAR, 1996; FANON, 1983; HARAWAY, 1995; HARDING, 1986/1996; LANDER, 2000; LUGONES, 2008; 
MIGNOLO, 2003; MOHANTY, 2008; OYĚWÙMÍ, 1997; SANTOS, 2010, entre muchos otros). El común denominador en estos esfuerzos de crítica se fundamenta en lo que se conoce como el argumento del ojo de dios, a saber, aquel que enuncia la pretensión supuesta en el proyecto de ver, nombrar, localizar, ordenar a todo lo demás en función de sí mismo, y, de todas formas, quedar sin ser visto en las descripciones y categorizaciones de las cosas del mundo. El problema de esta pretensión universalizante no se agota allí, se manifiesta especialmente en los efectos perversos que esta mirada ha tenido sobre los cuerpos, los territorios, las historias y los conocimientos nombrados y ordenados por este proyecto, en relación con este proyecto.

Se inventan con este ejercicio de nombrar vastas extensiones de "no ser" (FANON, 1983), o de ser en estos términos: no-civilizado, no-desarrollado, no-blanco, no-hombre, no-productivo, etc., y con esta invención, la necesidad de intervención, de colonización, de evangelización, de progreso. Es este modelo de racionalidad que sustenta todo este proyecto, lo que Boaventura de Sousa Santos va a empezar a denominar razón indolente. Para confrontar este modelo de razón indolente, Santos (2002) formula los prolegómenos de otra razón, una razón cosmopolita que, según él, tiene fundamentalmente dos tareas: por un lado, una tarea epistémica, de rechazar cualquier forma de epistemología general, por cuanto la diversidad epistemológica del mundo es potencialmente infinita, y compuesta por una multiplicidad de totalidades que no pueden, ninguna de ellas, ser subsumida o subsumir a otras. Por el otro, una tarea política, de encontrar nuevos sentidos de transformación social, de proyectos -con minúscula- de emancipación social, que puedan hacer frente a la operación del sistema de género moderno colonial capitalista.

La sociología de las ausencias pretende dar cuenta de la primera de las tareas de la racionalidad cosmopolita, a saber, la de ampliar el presente de modo que puedan aparecer como posibles, todas las formas de existencia que han sido negadas por la razón indolente. Este ejercicio de proliferación de totalidades parte de considerar la posición dominante de la ciencia moderna, para demostrar que lo no existente ha sido activamente producido como tal. Santos considera cinco lógicas de producción de ausencias:

Estamos así ante las cinco formas sociales principales de no existencia producidas o legitimadas por la razón eurocéntrica dominante: lo ignorante, lo residual, lo inferior, lo local o particular y lo improductivo. Se trata de formas sociales de inexistencia porque las 
realidades que conforman aparecen como obstáculos con respecto a las realidades que cuentan como importantes: las científicas, avanzadas, superiores, globales o productivas. Son, pues, partes descalificadas de totalidades homogéneas que, como tales, confirman lo que existe y tal como existe. Son lo que existe bajo formas irreversiblemente descalificadas de existir. (SANTOS, 2011: 32)

Mi intención en este texto es transformar las ausencias producidas por la ciencia de la paz en presencias, indagar sobre los modos en los que estas ausencias confrontan cada una de las lógicas de producción de ausencias, y se constituyen en alternativas creíbles a las experiencias hegemónicas. Esta constitución de las alternativas participa de la segunda tarea de la razón cosmopolita que realiza la sociología de las emergencias, a saber, la de investigar las condiciones concretas en las que estas alternativas pueden ofrecer pistas hacia el futuro.

Los estudios feministas de la paz (GILLIGAN, 1982; MANCHANDA, 2005 \& TICKNER, 2012) plantean que al mostrar la continuidad que existe entre las violencias más públicas y aquellas que acontecen en el ámbito de lo privado, como un ejercicio de producción de conocimiento desde las experiencias y las vidas vividas, y no como un asunto de Estado, aparecen opciones alternativas de significados de la paz que no están ya simplemente circunscritos a un modelo, sino que muestran los modos locales, cotidianos y situados en los que operan las violencias y por lo tanto muestran las pistas de lo que podría entenderse como unos sentidos posibles de paces plurales y distintas, situadas y concretas, pero paces al fin y al cabo para las comunidades y colectivos.

Es en este sentido que los feminismos terminan proponiendo una reconceptualización de la ciencia de la paz a propósito de la idea de cotidianidad que aquí retomo (MENDIA, 2014). No sólo porque descentran la descripción de la violencia y sus efectos del nivel más estatal y público, sino porque apuestan a una transformación de las condiciones que reproducen la violencia desde las experiencias, las resistencias y las alternativas que, especialmente las mujeres, construyen a los entornos violentos. Esta idea de transformación local, contextual y situada no sólo pone el énfasis de la conversación sobre la paz en las personas, colectivos y comunidades directamente afectadas por la violencia, sino que pluraliza los modos en los que comprendemos y construimos estas paces de la vida cotidiana, las paces en plural que pueden llegar a constituir los puentes y 
solidaridades entre las luchas por la vida digna de territorios y poblaciones históricamente marginadas de esta conversación.

\section{LA HISTORIA DE ESTE MAPA}

En el año 2014, en medio del proceso de paz entre el gobierno colombiano y la guerrilla de las FARC-EP, comienza la discusión en el Congreso de La República sobre la necesidad de reglamentar una cátedra obligatoria en las instituciones educativas sobre la paz. La Ley 1732 incluye los temas, asuntos, problemas y apuestas que, los diferentes grupos de investigación universitarios de las principales ciudades del país consultados por el Congreso de la República consideraron fundamentales para el aprendizaje de la paz por parte de los-as ciudadanos-as en formación en todo el país. Tanto la Ley ya citada, como el decreto 1038 expedido por la Presidencia de la República un año después crean los referentes de lo que las generaciones futuras entenderán por vivir y construir la paz.

Estos eventos en la construcción de la política pública son vistos desde mi posición en el Colectivo de Educación para la $\mathrm{Paz}^{2}$ como una emergente dicotomización del discurso: entre la paz institucionalizada y centralizada de la política pública, y la paz que estaba en proceso de discutirse en los contextos de práctica, en diferentes territorios, de los que participábamos los-as miembros del Colectivo. Es por esta razón, que me propuse estudiar el espacio-problema en el que está inmersa esta dicotomización para entender las lógicas en las que se produce la disputa por el significado de la educación para la paz, así como las lógicas con las que, al mismo tiempo, se invisibilizan y silencian sus alternativas.

Así fue como me propuse, por un lado, construir un archivo sobre los referentes fundacionales de la producción de conocimiento y política pública sobre educación para la paz, y por el otro, participar como co-coordinadora de un proceso de investigación-acción sobre las

\footnotetext{
2 "El Colectivo Educación para la Paz se constituye como un esfuerzo de distintas universidades, centros de investigación y organizaciones no gubernamentales del orden nacional, liderado, hasta la fecha, por más de 92 académicos y representantes de 40 instituciones públicas y privadas, por el diseño, gestión y ejecución de planes, programas y proyectos de impacto nacional e internacional orientados a disminuir prácticas sociales de exclusión y violencia, así como fortalecer una cultura de la paz, la convivencia pacífica y el reconocimiento de las diferencias." (Colectivo Educación para la Paz, 2017)
} 
prácticas pedagógicas en educación para la paz que adelantábamos en nuestros contextos con varios-as de mis colegas del Colectivo de Educación para la paz (SÁNCHEZ-CORRALES et al., 2020).

Para profundizar sobre las relaciones entre ambos sentidos de paz me propuse hacer un recorrido por el país para visitar los contextos de práctica de los-as investigadores-as/miembros del colectivo que estaban participando en la investigación colectiva. Estos espacios los escogí con base en unas entrevistas iniciales que hice a cada uno-a de los investigadores-as que accedieron a participar de este proceso, en las que pudieran profundizar sobre sus posicionamientos políticos con respecto al contenido y las estrategias de la educación para la paz. Cada uno-a de ellos-as me invitó a participar de sus contextos de práctica de diferentes modos; en ningún caso fui a observar solamente, al contrario, fui a participar activamente en la movilización de ejes de actuación política-pedagógica que cada uno-a de ellos-as requirió de mi parte en sus contextos.

Lo que presentaré a continuación es una síntesis de la investigación del espacio-problema en la forma de una cartografía de las ausencias y las emergencias que encontré en este proceso de indagación. Estas sociologías me permiten dar cuenta de las lógicas en las que son intencionalmente producidas como inviables las alternativas en las cuales, preguntando y conversando, yo encuentro potencialidades múltiples de resistencia y alternatividad a las violencias por las que atraviesan los contextos de los que pude participar.

\section{LAS AUSENCIAS}

Para abordar la primera de las tareas, la tarea epistémica de la sociología de las ausencias, me he propuesto partir de la posición dominante de la producción de conocimiento de la ciencia moderna, para buscar allí, las lógicas con las que estos discursos han producido activamente ausencias que tienen efectos materiales y cotidianos. Empezaré esta indagación en el campo de conocimiento conocido localmente - en Colombia- como la violentología, explorando en sus supuestos la manera en la que este relato sobre la Violencia en Colombia fue articulándose con discursos más globales y teniendo efectos locales. A partir del análisis de los efectos producidos por las ausencias construidas en este campo, me propongo analizar algunas de las materialidades 
de estos discursos en las cotidianidades de las prácticas que se han venido estudiando en esta investigación, en particular en las formas en las que estos discursos enuncian como ausencias, totalidades que resisten y se declaran alternativas a los mismos.

\subsection{PREMISAS EN FUNCIONAMIENTO}

La violentología se ha denominado a la gran cantidad de estudios que, especialmente sociólogos-as y politólogos-as, han hecho desde la década de 1960 sobre la violencia en Colombia (CHAMBERS, 2013; DEAS, 1999; GUZMÁN ET AL, 2005 \& SÁNCHEZ ET AL., 1985). La amplia mayoría de ellos-as tienen en común haber abordado el fenómeno desde un análisis de las potenciales causas explicativas de la violencia, es decir, desde los factores políticos, económicos, sociológicos y culturales que pudieron haber conducido a la multiplicidad de violencias en el territorio colombiano; con el objetivo, en parte, de diseñar estrategias que pudieran contrarrestar estos factores conducentes a la violencia y, de esta forma, generar condiciones para la consecución de la paz.

La producción de conocimiento sobre la violencia en Colombia a partir de sus causas con miras a su resolución tiene una abundante cantidad de efectos tanto en la toma de decisiones por parte de los gobiernos que asumieron la veracidad de estos informes, como en la puesta en marcha de una gran cantidad de estrategias para la intervención del conflicto colombiano por parte de la comunidad internacional. A continuación, me dispongo a analizar algunos de estos efectos, en particular en su contribución a la producción activa de ausencias que han justificado la intervención y explotación de territorios y poblaciones.

\subsubsection{SUPUESTO CULTURALISTA DE ENDOGENEIDAD}

El primero de los efectos de este análisis basado en las causas es que justamente se ha hecho exclusivamente con relación al contexto político, cultural, económico, histórico y geográfico colombiano. Es decir, todos los análisis suponen sin excepción que la violencia en Colombia tiene un origen endógeno y que, por lo tanto, las violencias de este modo producidas hacen parte de nuestra matriz cultural. Esta lectura endógena de la violencia no sólo naturaliza el orden violento del país, como si estuviera impreso en el ethos de la nación; sino que, 
adicionalmente, invisibiliza todas las continuidades que hay entre las violencias globales que operan localmente, aquellas vinculadas a las relaciones de explotación de recursos y poblaciones que han operado en el sur global como consecuencia del colonialismo, el capitalismo y el heteropatriarcado.

Al ubicar geográficamente el origen específico y exclusivo de la violencia en Colombia, éste se vuelve un problema colombiano, que debe ser solucionado localmente, y que por tanto autoriza diversas formas de intervención internacional para alcanzar la estabilidad y viabilidad de las instituciones que el país no ha alcanzado por su cuenta. Esta dinámica de relacionamiento alrededor de un problema local, no sólo es altamente productivo en términos de las estrategias que local e internacionalmente se disponen para su resolución, sino también de los discursos que justifican toda clase de intervenciones a un Estado que ha mostrado ser incapaz, insuficiente e, inclusive, fallido.

\subsubsection{LA AUSENCIA DEL ESTADO}

Pero inclusive esta idea de la ausencia del Estado, vista por varios de los estudios fundacionales de la violentología como causa objetiva de la guerra en Colombia (OQUIST, 1978 \& PÉCAUT, 2012), oculta las formas en las que esta idea de ausencia ha permitido una relación de explotación entre el centro metropolitano y sus periferias. Es decir, es gracias a esta ausencia del Estado que el modelo económico ha logrado generalizarse a todo el territorio colombiano, configurando un modo de operación en el que las periferias han estado disponibles para su explotación por parte de las élites locales y sus correlatos globales:

A partir de esta idea, el problema se reduce a la ausencia del Estado, al hecho de que no se ejerce plenamente la soberanía. Esta ausencia ha mostrado ser, sin embargo, más que una expresión de la debilidad o de la disfuncionalidad del Estado (incapacidad, baja cobertura, corrupción, etcétera), la forma particular que ha caracterizado históricamente su acción frente al conjunto de realidades que ha sido considerado como fronteras internas: el país anclado en el pasado y en el desorden, que espera ser redimido por la llegada del progreso para poder así ingresar a la nación y hacer parte de la historia. El concebir las realidades fronterizas de manera autorreferencial, como externas a la nación, e incluso como opuestas a ella, impide reconocerlas como producto de un mismo devenir, de un mismo contexto: ha contribuido a su reificación, al tiempo que oculta los procesos mediante los cuales se establecen y se decretan su diferencia y su separación. Por lo demás, desde la perspectiva de su relación con la periferia resulta inocultable que el proyecto nacional se 
ha visto reducido a su incorporación a la economía metropolitana, es decir, a los sistemas económicos y militares necesarios para garantizar la explotación intensiva y extensiva de sus poblaciones y paisajes. (SERJE, 2011: 308)

Además de ocultar los modos en los que este ordenamiento político ha contribuido a profundizar la operación del sistema económico, esta noción de la violencia vista como ausencia de Estado, refuerza la idea de que es con la expansión de la modernidad liberal y su expresión material en instituciones estatales que lograremos alcanzar la tan anhelada paz. La tesis de Jiménez-Ocampo (2014) es que desde la promulgación de la Constitución Política de 1991 se empieza a realizar la tarea de fortalecimiento institucional estatal que llevaba siendo señalada por todos los estudios de la violentología -tanto locales como foráneos- como la fórmula para la consecución de la paz.

Así, la tesis de la ausencia del Estado invisibiliza tanto el sistema económico en operación en la explotación de las periferias, como los intereses creados por las élites políticas en la administración de la guerra como estrategia de reproducción de ese mismo sistema. Esta idea de vacío es activamente productora de las condiciones por las cuales se justifica y sostiene tanto el estado de excepción con el que se administra la guerra, como las continuadas intervenciones sobre los territorios y poblaciones "desocupadas".

\subsubsection{LA ECUACIÓN QUE IGUALA PAZ A DESARROLLO}

Las intervenciones arriba mencionadas no sólo asumen la forma de la explotación minera y agroindustrial por parte de las grandes corporaciones que tienen en estos espacios vacíos toda la impunidad para garantizar de forma ordenada la explotación y la extracción con miras al crecimiento económico; sino también, se manifiestan en la presencia diferencialmente distribuida en estos territorios vacíos de los "chalecos", funcionarios de las agencias de cooperación internacional que llegan a las zonas de mayor incidencia del conflicto a través de programas de gobierno, y estrategias para el desarrollo.

Lo particular de esta perspectiva es que el desarrollo, como prescripción para la paz, abandona muchas de las demandas estructurales hacia el cambio de asuntos que solamente se pueden transformar en el nivel macroeconómico, para apostar nada más que por la dimensión de lo local. Así pues, los territorios de la violencia que antes eran anómalos por 
su condición de lugares de la guerra, ahora lo son porque se constituyen en lastres de dinámicas más intensivas de desarrollo. (JIMÉNEZ-OCAMPO, 2014: 120)

Es a través de estos mecanismos que se buscó reemplazar el deseo de cambio político emergente en algunas de las zonas de influencia guerrillera, por el deseo de desarrollo. De esta forma, se empezaron a deslegitimar discursivamente los argumentos que sostuvieron por mucho tiempo la lucha armada de las guerrillas, para ser reemplazadas por demandas locales de servicios que entraban en el paquete del desarrollo. En este movimiento, el discurso de los Derechos Humanos, así como los talleres de capacitación para el desarrollo fomentados por las agencias de cooperación internacional fueron fundamentales.

De esta forma, el discurso que iguala paz a desarrollo no sólo invisibilizó el papel que juegan los asuntos estructurales en la dinámica de la violencia en Colombia; sino que logró ubicar la solución a esta violencia en el ajuste progresivo de las condiciones para el desarrollo en espacios específicos, con relación a los comportamientos y voluntades de sujetos específicos. Así, se volvió tarea de cada uno de los ciudadanos del Estado, aprender unas ciertas capacidades para el desarrollo, de modo que progresivamente fueran alcanzando las condiciones de vida y dignidad que los habían mantenido al margen de la paz, individualizando la responsabilidad en la solución de los problemas económicos y sociales.

\subsection{EFECTOS DE ESTAS PREMISAS EN LAS COTIDIANIDADES DE LAS PRÁCTICAS}

Este aparato conceptual sobre cómo entender las causas de la violencia y, por ende, los mecanismos de construcción de la paz han producido una multiplicidad de ausencias, es decir, han puesto en circulación relatos sobre la paz que declaran inviables, no-existentes o inferiores apuestas alternativas que emergen de las experiencias y cotidianidades de comunidades que han resistido las violencias por no responder de manera estructural a estos diagnósticos más científicos. A continuación, me propongo revisar procesos de constitución de estas ausencias en varios de los contextos de práctica de los que tuve la oportunidad de participar en el proceso de investigación colectiva. 


\subsubsection{PAZ COMO DESARROLLO}

A partir de este tema de las experiencias le planteo en forma de paradoja el tema de la posición ambigua que jugamos como activistas de este tema de la paz en nuestras instituciones, porque conociendo las experiencias, nos hemos dado cuenta de que la paz está sirviendo para que el gobierno deje entrar los proyectos de los grandes negocios de las multinacionales, y que, sin embargo, asumimos la responsabilidad de defender el proceso de paz como lo más razonable en momentos como este. Ella comenta a partir de esta paradoja que para ella fue muy significativa la experiencia de Guatemala, en el sentido en que las comunidades que se visitaron reportaban todas ellas que con la paz vino el desarrollo, el desarrollo entendido como la ocupación y explotación de parte de las multinacionales de sus territorios. Me cuenta que esta es la aproximación que se tiene desde muchas carreras. A propósito de la cátedra de paz en la universidad, han tenido la oportunidad de hacer conversatorios con profesores, y me dice que por ejemplo los de turismo no problematizan este tema, al contrario, piensan en la paz como una oportunidad para ampliar los espacios de ocupación de la industria hotelera en zonas en las que no había acceso, los antiguos territorios en conflicto. Me cuenta también, que los de salud, piensan en la oportunidad que ahora tienen de acceder a los cuerpos de los guerrilleros como depositarios de una gran cantidad de antígenos contra enfermedades tropicales que se pudieron haber desarrollado fruto de su permanencia en la selva.

Antes de empezar la clase, Luz tiene en sus manos el libro de Eduardo Galeano "Patas arriba", lo abre en una página doblada por ella, me lee un párrafo, y me dice: a propósito de nuestra conversación sobre el desarrollo. El párrafo dice lo siguiente:

El desarrollo

El puente sin río.

Altas fachadas de edificios sin nada detrás.

El jardinero riega el césped de plástico.

La escalera mecánica conduce a ninguna parte.

La autopista nos permite conocer los lugares que la autopista aniquiló.

La pantalla de la televisión nos muestra un televisor que contiene otro televisor, dentro del cual hay un televisor.

(Diario de campo, Medellín, Marzo 13 de 2017)

Luz es profesora universitaria en Medellín y activista del movimiento de derechos humanos de Antioquia. Ahora coordina en su universidad la Cátedra de paz, y es esta la experiencia que ha sistematizado junto con el colectivo de investigadores-as. Mi participación en este espacio fue de colaboración en una de las actividades que estaba programada para la cátedra en la universidad. 
En ese contexto tuve ocasión de compartir y discutir con los-as estudiantes de diferentes programas académicos. La conversación a la que hace referencia la viñeta plantea al menos tres asuntos clave sobre las ausencias producidas en este proceso de educar para la paz.

El primero de ellos tiene que ver con la paradoja del activismo a la que se hace referencia al comienzo, pues por un lado está el imperativo ético-político del activismo y de la educación de asumir la responsabilidad de cara a la coyuntura y defender públicamente la opción ética de la paz. En uno y en otro caso hay un carácter normativo de la práctica que supone tomar partido y definir el curso de acción en función de aquello que reconocemos como la mejor de las opciones disponibles. Sin embargo, tenemos, por el otro lado, una gran cantidad de motivos y experiencias previas, conversaciones cercanas con quienes han sido más afectados por la violencia, que nos están indicando que una vez más, la promesa de la pacificación está siendo utilizada para abrir fronteras al desarrollo.

El segundo asunto tiene que ver justamente con esto. En octubre de 2016 participamos junto con Luz y otros-as integrantes del colectivo en un evento que tuvo lugar en los territorios de comunidades indígenas guatemaltecas para escuchar sus impresiones acerca del balance de los 20 años de la firma del acuerdo de paz en ese país. Para todos-as era claro que la paz sólo había significado desarrollo en términos de la entrada del gran capital a la explotación de sus recursos naturales; nada había tenido que ver con las reivindicaciones por reconocimiento y soberanía que llevaban exigiendo al Estado por décadas. Fue en estas conversaciones que nos dimos cuenta de las profundas continuidades que hay entre las violencias que ocurren en Nuestra América, de la operación local de un sistema global que, aunque tiene particularidades en cada territorio, no se fragmenta por las fronteras abstractas de los estados, sino de las periferias producidas como disponibles para su explotación.

Finalmente, el tercer asunto que reclama atención en esta idea de paz como desarrollo es la forma en la que es incorporada la paz en el funcionamiento de la ciencia moderna. El relato de los-as estudiantes sobre los usos estratégicos de la paz para el desarrollo de sus respectivos campos disciplinares llama mucho la atención. No sólo por la expansión del turismo como resultado de la apertura de fronteras, sino especialmente por la instrumentalización de los cuerpos 
de los-as excombatientes. Aquí ellos-as son vistas como objeto de investigación y extracción, jamás como sujetos de conocimiento y experiencia.

La paz como desarrollo pone en circulación un orden posible, del que participan la ciencia y el capitalismo como estrategias ineludibles en el proceso de su construcción. Así, se sedimenta un sistema de valores que designa como avanzado, superior, científico y mejor todas las opciones que estén codificadas en estos mismos términos. Aquellas que no han sido aún traducidas a esta lógica están condenadas a desaparecer.

\subsubsection{DESAPARICIÓN POR CIUDADANÍA}

Marta ${ }^{3}$ interviene a continuación diciendo que hay muchas formas en las que se ejerce la violencia en la universidad. No sólo de estas formas explícitas de las que ha hablado Pedro, sino por ejemplo, en las expectativas que les imponen de sus ejercicios profesionales. En su caso, como estudiante de una tecnología en finanzas, desde el primer semestre le exigen que tienen que formar una empresa. Ella, al comienzo trató de obviar esta parte, pero progresivamente en la carrera se volvió cada más apremiante. Ella trató de explicar en la universidad que ella estudiaba ese programa para volver al resguardo y usar ese lenguaje para ayudar a su comunidad a gestionar recursos con programas del Estado, gestión a la que no habían tenido acceso justamente porque nadie allá sabía de esas cosas. Así que ella asumió esta responsabilidad por su comunidad, pero ella no quería formar empresa, porque esto iba en contra de su cultura, de sus tradiciones y de su retorno. Esta violencia, dice ella, es mucho más sutil, pero en el fondo más fuerte, son este tipo de prácticas que hay en las universidades las que se usan para exterminar nuestra cultura, para que los estudiantes indigenas que entran, progresivamente se vayan perdiendo con el paso de los semestres, primero por la presión social de la discriminación por ser diferentes, y luego porque se vuelven ciudadanos y nunca regresan al resguardo.

(Diario de campo, Neiva, Abril 5 de 2017)

Henry es profesor e investigador en una universidad en Neiva. Su investigación ha estado ligada al acompañamiento y seguimiento de la conformación del Cabildo Indígena Universitario, y es justamente esta la experiencia que ha decidido sistematizar junto con el colectivo de investigadores-as. Fui a Neiva a participar de uno de los coloquios que Henry organiza junto con los-as estudiantes del Cabildo cada semestre para intercambiar experiencias. En esta ocasión tuve la oportunidad de conversar con estudiantes que han migrado desde diferentes resguardos de la

\footnotetext{
${ }^{3}$ El nombre ha sido cambiado para proteger la identidad de la estudiante

${ }^{4}$ El nombre ha sido cambiado para proteger la identidad del estudiante
} 
región para estudiar en la universidad pública gracias a la ley de cuotas. En estas conversaciones apareció esta idea de la desaparición por ciudadanía que ya había escuchado antes en una visita a un colectivo de mujeres campesinas del Sumapaz.

Tanto en esa ocasión como en esta, se identifica la idea de ciudadanía como una producción de deseo muy fuerte hacia los miembros más jóvenes de las comunidades, ya sean campesinas o indígenas. En el caso de las mujeres campesinas, ellas se identificaban como protectoras del páramo, y por esta condición, se sentían muy preocupadas por la continuidad de esa función. No sólo por la creciente presencia de explotaciones mineras, sino porque los-as más jóvenes de la región codificaban sus expectativas de vida hacia el futuro en términos muy urbanos: ir a la universidad, conseguir un trabajo, tener un salario; y muy pocos en términos de permanecer en el territorio y cuidar del páramo.

En el caso de los-as estudiantes indígenas, en particular, en el caso de Marta, no es ella quien desea tener la vida urbana; se trata más bien de una imposición epistémica de su carrera profesional, de formarla en unas capacidades que le permitan participar activamente en las instituciones sociales, políticas y económicas de la ciudad. Hacer empresa, vestir de un cierto modo, utilizar un cierto repertorio de vocabulario, desear progresar y ganar dinero, son todas capacidades que parecen imponerse como condiciones epistémicas para participar de la vida de la universidad.

Aunque la expresión de ciudadanía está siendo usada aquí en al menos dos sentidos diferentes, por un lado, el que aparece de forma implícita en la viñeta sobre las condiciones que permiten participar de las instituciones sociales y políticas de la vida en la ciudad; y por el otro, el que se pone en circulación en el discurso de educar a la ciudadanía; me gustaría argumentar aquí que ambos participan de la misma matriz de producción de deseo.

Esta producción de deseo - que es reforzada por las instituciones educativas y los conocimientos que reproducen- pone en circulación un sistema de valores relacionados con una forma de vida posible, hegemónica y con la pretensión de ser homogénea. Se educa a los estudiantes para participar de una serie de instituciones sociales que, al menos en nuestro contexto, adquieren forma en la vida de la ciudad. Se trata de una educación que presupone que 
los individuos que participan de ella deben llegar a alcanzar unos estándares de vida definidos de antemano como el acuerdo social sobre la vida buena: vivir en democracia electoral y participar exitosamente de la economía de mercado.

El problema con esta definición prefabricada de vida buena radica en que educar para ser ciudadanos-as de esta forma implica dejar de hacerlo para ser de otros modos, y al mismo tiempo, implica educar para no querer ser de otros modos, para desvirtuarlos como alternativas posibles, legítimas de vivir en el mundo.

\subsubsection{ESTA NUEVA GUERRA QUE ES LA PAZ}

El coloquio empieza con la presentación de Juan ${ }^{5}$, tanto del CRIHU como del programa de Educación y Cultura del Consejo. El trabajo del CRIHU consiste en la reivindicación $y$ defensa del territorio, y entienden la paz como sinónimo del "buen vivir" que consiste para ellos en la garantía de "tierra, cultura y autonomía". En este momento, lo interrumpo para preguntarle cómo ha percibido el proceso de paz y su implementación, a lo que me responde con otra pregunta: “¿cuál paz? si en el momento en que este actor armado se concentró en las zonas veredales, al día siguiente a nuestros territorios ya habian llegado otros actores armados reclamando soberanía. No me malentienda, nosotros hemos apoyado el proceso, y nos parece importante alcanzar acuerdos con los grupos armados, pero las violencias son tantas, que todavía hay mucho por hacer, de hecho, los pueblos tanto indigenas como afrodescendientes tuvieron que luchar mucho para ser incluidos en un capitulo étnico que permitiera garantizar los derechos hasta ahora alcanzados. Sin embargo, el tema territorial nos preocupa, porque con esta firma no se va a garantizar la paz, ya han asesinado a 120 líderes sociales, seguimos estando en conflicto, sólo que cambiaron los actores, tenemos situaciones nuevas que generan nuevas guerras. Hay una politica de estigmatización contra nosotros, todos los privilegios que hemos conseguido han sido a punta de lucha y movilización, poniendo muertos y desaparecidos. $Y$, sin embargo, hasta ahora no hay una politica, ni siquiera esta de la paz, que nos incluya, de hecho muchos de los programas están en nuestra contra."

(Diario de campo, Neiva, Abril 5 de 2017)

Cuando Juan planteó el asunto de la paz en el Coloquio que había organizado Henry en Neiva en estos términos pensé inmediatamente en las narrativas de los campesinos colonos del alto Ariari que recoge Alfredo Molano en la voz de uno de sus entrevistados: “...Eso fue antes de la paz; de la medio paz que hubo cuando Pinilla y antes de la otra paz que hubo cuando cayó.

\footnotetext{
${ }^{5}$ El nombre ha sido cambiado para proteger la identidad del participante.
} 
Porque ha habido tantas paces como guerras. Muchas. La paz ha sido la disculpa para que vuelva a tronar la guerra" (MOLANO, 2010: 191).

Esta paz parece ser una nueva guerra en otros términos, en unos términos diferentes a los que se habían tenido hasta ahora, en unos términos para los que aún no se han inventado estrategias de lucha, de resistencia. Esta nueva guerra que es la paz en una expresión que escuché en una visita a una comunidad de paz, justamente en el Alto Ariari, cuando hacían un balance de la situación en la que se encontraban ahora que se había concentrado la guerrilla de las FARC-EP en las zonas veredales. Una de las lideresas de la comunidad decía que en esta nueva guerra se habían cambiado las balas por licencias ambientales, y que este nuevo escenario, la lucha posible estaba ahora ligada a la defensa del agua y del territorio de las grandes empresas que estaban llegando para hacer explotación minera en las zonas a las que no habían podido acceder antes por efecto de la presencia guerrillera.

Esta nueva guerra que es la paz preocupa a campesinos y comunidades indígenas, y en particular a Juan, porque el marco general desde donde se enuncia vuelve a poner en cuestión asuntos que ya se habían pactado entre el Estado y las autoridades indígenas sobre la autodeterminación y la soberanía en sus territorios. Las aperturas que contiene el acuerdo pueden derivar o en una mayor autonomía de los pueblos sobre sus formas de vida; o en un acceso total a sus territorios de las empresas multinacionales y los intereses del gran capital.

Esta apertura, en un contexto de acuerdo que asume la paz como desarrollo como veíamos en la primera de las categorías, como democracia electoral más economía del mercado, las posibilidades de estas otras formas de habitar el territorio, de significar la existencia, de defender alternativas otras de vida son cada vez más escasas, más amenazadas directamente que incluso cuando la guerra se hacía con desapariciones y masacres.

Lo interesante de la expresión justamente radica en que pone en el mismo nivel, en la misma posición amenazante, tanto a la guerra, en tanto constituye una amenaza directa contra sus vidas, como al proyecto de paz liberal, en tanto constituye una amenaza a la forma de comprender la vida en el territorio. En el primer sentido, la amenaza es contra las vidas 
individuales de quienes hacen parte de la comunidad; en el segundo sentido, la amenaza es contra el sentido que tiene la vida de la comunidad.

\subsection{EN SÍNTESIS}

Hemos visto hasta aquí varias formas en las que opera la producción activa de ausencias en el campo de conocimiento producido a propósito de la violencia y de la paz, así como sus correlatos en las cotidianidades. Este ejercicio de sociología de las ausencias ha servido para argumentar que las pretensiones actuales sobre la construcción de la paz generan una multiplicidad de violencias adicionales, específicamente sobre quienes se han visto en mayor medida afectados por las violencias. En este caso, a partir de tres de las cinco lógicas enunciadas por Santos (2006), a saber, las monoculturas del saber, del tiempo lineal y de la escala dominante.

Con respecto a la primera lógica, la monocultura del saber, vimos cómo los conocimientos que circulan en la universidad, así como las prácticas que los sostienen y legitiman se imponen sobre cualquier otra forma de conocimiento, o usando estos otros lugares de producción de conocimiento como objetos de estudio o campos de experimentación. Con respecto a la segunda lógica, la del tiempo lineal, vemos los efectos que comunidades de diferentes regiones reportan sobre la automática apertura de sus territorios a los grandes proyectos de explotación agroindustrial y minero-energética, garantizada por los grupos armados paramilitares encargados con la tarea de abrir materialmente estas fronteras al desarrollo. Y con respecto a la tercera lógica, la de la escala dominante, vimos la desaparición a la que están condenadas poblaciones que organizan el sentido y la cotidianidad en coordenadas diferentes a las de la ciudadanía; su desaparición por efecto de la educación de ciudadanos que se emprende como modelo universal de vivir en el mundo no sólo ocurre en las instituciones educativas, sino en las operaciones del sistema capitalista en su fase neoliberal que cada día cierra más las posibilidades de la vida en el campo.

Agregaría yo una lógica de producción de ausencias que se ve efectivamente puesta en marcha no sólo en este contexto colombiano, sino que se reporta en lo que desde el 2001 se ha llamado "la guerra contra el terrorismo", a saber, la producción de ausencias vía su 
deslegitimación por "peligroso", condición que construye la necesidad de intervención en estos territorios y poblaciones para "pacificarlos". Aunque esta lógica está estrechamente ligada a la del desarrollo, es diferente por cuanto a los depositarios de esta ausencia no se les considera simplemente pobres, o desposeídos, o negados, sino activamente peligrosos para ellos mismos, para los otros, y para el gran proyecto de la paz y la seguridad. Por esta razón, la intervención que se hace en estos territorios no es sólo a través de los diferentes modos de gubermentalidad, sino por la vía de la fuerza, las armas y la violencia.

\section{LAS EMERGENCIAS}

Pero la intención de esta cartografía no se agota aquí, en el diagnóstico o su descripción desde nuevas categorías analíticas; al contrario, el objetivo es ahora explorar en estas presencias que logramos ubicar en el mapa luego de haber sido intencionalmente producidas como ausencias, pistas de alternativas a las experiencias hegemónicas. Estas pistas emergieron justamente en los contextos de práctica en los que, y a propósito de la conversación sobre la educación para la paz, diferentes comunidades, en diferentes territorialidades apuntan condiciones y posibilidades en las que, efectivamente, pudieran emerger contenidos, plurales y situados, de ideas de paces posibles.

Sobre este asunto de las alternativas quiero detenerme. Porque ha sido amplia la literatura, especialmente la heredera de Foucault (1988), la que ha propuesto nombrar estos espacios de alternatividad como resistencias al ejercicio de los poderes hegemónicos. Hay, sin embargo, una discusión aquí urgente con respecto a lo que implica para esta cartografía sobre el ejercicio del poder del sistema de género moderno colonial capitalista en el contexto de la educación para la paz en Colombia asumir la resistencia entendida en estos términos. Implicaría, entre otras, que la resistencia no es más que la reafirmación de la inevitabilidad y universalidad del Proyecto moderno colonial patriarcal capitalista (MENESES, 2016).

Es por esta razón que a continuación me gustaría detenerme en las pistas y señales que emergieron en los contextos de práctica del proceso de investigación como opciones de lucha, alternatividad y solidaridad, tanto a la operación de las múltiples violencias directas y cotidianas, 
como a los grandes proyectos económicos, políticos y epistémicos -como lo es el de la construcción de paz- que han pretendido invisibilizarlas como posibilidad. Creo que, aunque en el caso de este proceso de investigación fueron relevantes las respuestas novedosas y creativas a la violencia, en tanto respuestas, también es importante revisar en ellas las aperturas que operan en la diferencia entre re-existir y sobrevivir al conflicto.

\subsection{ORGANIZARSE PARA HACER LA TRADUCCIÓN}

Hablando con Henry, me cuenta que todo el proceso con el Cabildo Indígena Universitario comienza con una exploración inicial en la Universidad para identificar las problemáticas de estos grupos que habian ingresado en el proceso de la ley de cuotas. En medio de esta exploración fue que se encontraron con una organización que venía desde el año anterior al comienzo de la indagación, desde el 2012. Me dice Henry que eran "jóvenes que estaban dentro de una universidad politizándose, colectivizándose en un cabildo indigena universitario, que tenía unas condiciones y una estructura política propia de los resguardos. Entonces, todo eso me llama la atención, cómo las organizaciones sociales se mueven no solamente en la comunidad, dentro de las comunidades rezagadas también por el Estado. Es decir, me causó inquietud cómo las organizaciones sociales no sólo se gestan en lugares en donde el Estado más las rezaga, que es en las comunidades alejadas de lo urbano; sino cómo dentro de una unidad institucional, como lo es la universidad, se colectivizan los mismos jóvenes que sienten rezago, pero que el estado supone que están en las mismas condiciones de desarrollo. Todas estas formas de organizaciones que, además en algún momento cuando buscamos bibliografía, encontramos que habian sido poco abordadas."

(Diario de campo, Neiva, Abril 5 de 2017)

La sorpresa de Henry al encontrar procesos de apropiación y resignificación del espacio universitario por parte de estos-as jóvenes indígenas da cuenta de una de las pistas de acción política que emerge del proceso. En este caso se trata de una instancia de organización social-política-cultural de los-as estudiantes en la que no sólo se acompañan en el proceso de adaptación a la universidad, sino desde la que establecen un lugar de enunciación que la institucionalidad no puede simplemente ignorar o colonizar. Es decir, con la organización de su propio Cabildo al interior de la universidad están constituyéndose como un lugar de saber, de posicionalidades políticas, y de prácticas sociales y culturales.

Los cabildos indígenas universitarios en Colombia tuvieron un primer referente en la Universidad del Cauca, y desde allí han ido influenciando procesos organizativos de jóvenes en 
diferentes universidades públicas del país con el fin de crear un espacio para sus saberes y prácticas ancestrales dentro de la universidad, dentro de los rituales y lugares de la universidad, al lado de la ciencia moderna, en las clases, en los salones, pero también, en la vida cotidiana y universitaria. Para estos-as jóvenes de la Universidad Surcolombiana, ocupar el espacio ocurre tanto en la posibilidad de dar clases de lengua indígena como parte del plan de estudios, hasta tener sus propios espacios en el calendario académico para compartir con la comunidad universitaria prácticas y saberes venidos de sus propios resguardos.

La existencia de estos cabildos implica que los-as jóvenes indígenas que llegan a la universidad, llegan colectivizados, y en tanto colectivo reclaman espacios simbólicos para sus saberes que no pueden ser simplemente ocupados o colonizados por el conocimiento disponible en la ciencia moderna; Más bien, que están ahí para que se abra la conversación, y así como su participación en este espacio implica haber dado el paso al frente para hablar el lenguaje del Estado y el mercado, también exigen de las mayorías acercarse a hablar estos otros lenguajes, a emprender el trabajo de traducción:

El trabajo de traducción tanto puede darse entre saberes hegemónicos y saberes no-hegemónicos, como puede ocurrir entre diferentes saberes no-hegemónicos. La importancia de este último trabajo de traducción reside en que solo a través de la inteligibilidad recíproca y consecuente posibilidad de agregación entre saberes no-hegemónicos es posible construir la contra-hegemonía. (SANTOS, 2006: 95)

Este trabajo de traducción del que habla Santos implica una instancia de performatividad de las ecologías de saberes, en donde no sólo está presente el supuesto epistémico de que la diversidad epistemológica del mundo es potencialmente infinita, sino que está en juego además la práctica política que implica la labor de la traducción intercultural entre saberes venidos de matrices culturales diferentes. Este trabajo de traducción es el que creo está en funcionamiento en estos Cabildos Indígenas Universitarios; es el intento por poner en conversación dos lenguajes que no están en la misma posición de poder, en un espacio que además no es neutral frente a ambos, pero que los colectivos organizados para la traducción pretenden subvertir con prácticas estratégicas de ocupación simbólica de estos lugares y rituales. 


\subsection{LA VERDAD, LA MEMORIA}

Luz toma el libro de Eduardo Galeano "Patas arriba", lo abre en una de las páginas que tiene dobladas por las esquinas y empieza su clase leyendo lo siguiente:

\section{La memoria rota}

A fines del siglo dieciocho, los soldados de Napoleón descubrieron que muchos niños egipcios creían que las pirámides habian sido construidas por los franceses o por los ingleses.

A fines del siglo veinte, muchos niños japoneses creían que las bombas sobre Hiroshima y Nagasaki habian sido arrojadas por los rusos.

En 1965, el pueblo de Santo Domingo resistió durante ciento treinta y dos noches la invasión de cuarenta y dos mil marines norteamericanos. La gente peleó casa por casa, cuerpo a cuerpo, con palos y cuchillos y carabinas y piedras y botellas rotas. ¿Qué creerán, de aquí a un tiempo, los niños dominicanos? El gobierno no celebra la resistencia nacional en un Día de la Dignidad, sino en el Día de la Confraternidad, poniendo un signo de igual entre quienes habian besado la mano del invasor y quienes habian puesto el pecho a los tanques.

A continuación, Luz pone un video sobre la memoria. Se trata de un cuento narrado a dos voces, con intervenciones de transeúntes en Medellín. La historia se desarrolla en una ciudad de eterna primavera a la que un día llega un mercader ofreciendo dinero y joyas a la gente que acepte venderle sus malos recuerdos. En ese momento, la imagen captura a transeúntes que enumeran sus malos recuerdos: la violencia, el desplazamiento, las masacres, los homicidios, las bombas, el secuestro, etc. El cuento continúa narrando que la gente se aproximó masivamente al mercader y le vendieron todos sus malos recuerdos, todos excepto un hombre anciano. El mercader se fue de la ciudad y nunca nadie más volvió a saber de él. Los primeros días después de eso, la gente volvió a dormir tranquila y a experimentar una calma que nunca habian sentido. Pero con el tiempo, se dieron cuenta de que sus recuerdos hacian parte de lo que ellos eran, que eran los motivos de nostalgia, de felicidad y decidieron todos ir a buscar al único anciano de la ciudad que no habia vendido sus recuerdos para que les contara ese pasado que compartian y que los hacía ser quiénes eran.

(Diario de campo, Medellín, Marzo 13 de 2017)

Este relato sobre el contenido de la clase de Luz, de la cátedra de paz, contiene el potencial de activación política implícita en los ejercicios de hacer memoria. Las políticas de la memoria no sólo han posibilitado la multiplicación de los sujetos que son reconocidos como sujetos autorizados para contar la verdad de lo que pasó en décadas de violencia en Colombia; también 
ha puesto el énfasis de los estudios de la violencia y de la paz en la cotidianidad de las vidas vividas de los pueblos, de las organizaciones, de las comunidades, de las mujeres, de los niños-as.

El desplazamiento epistémico que se opera con la vuelta sobre lo múltiple y lo cotidiano tiene además consecuencias en los activismos políticos. Porque al hacer las violencias y las paces plurales, diferentes entre sí, apropiables a relatos y lugares próximos, a los cuerpos y a los nombres conocidos, también se activan las posibilidades de organización y movilización, y de rechazo colectivo a quienes directamente han operado el despojo y la opresión.

Así como Luz en su clase hace alusión a las memorias rotas con las que recordamos La Historia, también reivindica los lugares cercanos con los que hacemos y tejemos las memorias. Esta aparente dicotomía entre la singularidad del metarrelato, y la pluralidad de pequeñas historias es relevante por cuanto tiene que ver con las formas específicas con las que elaboramos versiones de nosotros mismos, como en la historia del mercader de recuerdos. Tiene que ver, como decía uno de los participantes al coloquio político del IPC -Instituto Popular de Capacitación- del que participé por invitación de Luz y Ariel, con que "no basta con decir quién mató a quién, es necesario que se establezca por qué los mataron y a quién benefició, porque hoy en día se pueden hacer hidroeléctricas y explotaciones mineras a manos de multinacionales gracias a esas matanzas."

Estas verdades históricas construidas por efecto de trazar estas continuidades entre la operación local de las violencias y los intereses económicos del gran capital, han logrado movilizar, especialmente en los últimos meses, a una diversidad de movimientos $\mathrm{y}$ organizaciones sociales, de las de siempre, y de las que empiezan a articularse a las luchas que son las mismas en diferentes territorios, rurales y urbanos, para denunciar en el espacio de lo público la veracidad de estas verdades.

Por eso la memoria está rota, por ser un lugar de disputa en el que se construyen versiones del pasado que arrojen luz para comprender el presente y las opciones de futuro (TROUILLOT, 1995). En este lugar de disputa tienen un rol hegemónico las versiones que el Centro Nacional de Memoria Histórica ha intentado ordenar como parte de una historia oficial y legítima del pasado. Las mismas que hoy, y como efecto de la reglamentación de la cátedra de paz, constituyen objeto 
de aprendizaje en las instituciones escolares. Esta función es reminiscente del mal de archivo que denuncia Derrida:

Recordando la obra de Derrida, Mal de Archivo (1995), la autora se preguntará pues por la forma en la cual la violencia del archivo sutura esta disyuntiva, sutura las fisuras de la memoria al imponer su ley, entendida como la de la membresía a la comunidad por medio de la repetición de la memoria. También diría que el archivo para Derrida envuelve pues la operación hermenéutica que ya trabaja asumiendo objetivos explícitos: la totalización, la unificación del poder político y la eliminación de lo heterogéneo. (APARICIO, 2016: 5)

Al suturar con su oficialidad las otras memorias e imponer su totalidad como la versión completa y disponible sobre el pasado, se desplazan y cancelan estas otras versiones que, como la del IPC, ordenan la verdad histórica -no epistémica- en función de las activaciones políticas, de solidaridades que pueden emerger en virtud de localizar en el cuerpo y en el territorio la continuidad entre las violencias y sus imperativos de justicias posibles.

Aquí, lo que se produce como ausente no son sólo estas verdades otras, y sus respectivos imperativos de justicia; lo que implica esta pretensión de ordenar una sola versión el pasado es el activo proceso de ocultamiento de todo lo que arsenal de violencia quiso borrar como posibilidades de organización política, social y económica. Son las huellas de estas otras verdades y estas otras justicias las que hoy, en los relatos sobre el pasado, nos arrojan pistas sobre proyectos futuros.

\subsection{RESIGNIFICAR LAS HERRAMIENTAS}

Después de esta intervención, viene una introducción al espacio, de parte del observatorio del IPC, quien apela al acervo que de hecho ya existe en el movimiento social para poder comprender este momento de verdad. Dice, sin embargo, que la tarea que emprenden desde hoy, supone resignificar los derechos, por lo menos la idea asociada a ellos que viene de occidente y según la cual son esencias evidentes, atemporales, universales para pensar en mecanismos de diálogo intercultural que rompan con la lógica en la cual se ha dado una gestión controlada de la vida, en la que han servido como dispositivos jurídico-politicos usados para explotar la vida, en función de un discurso de seguridad que ha entrado en tensión con la libertad, y que ha privilegiado la seguritización como derecho humano. Este discurso se identifica con los esfuerzos de la derecha. Hay ahora una necesidad de resignificar los derechos para rescatar su potencial emancipatorio desde las prácticas contrahegemónicas de la resistencia. Concretamente, hay que desencializarlos, desmercantilizarlos, instrumentalizarlos, de modo que no sean totalizantes, sino que sirvan para politizar, para hacerlos planes de gobierno. Se concluye que es necesario para este grupo 
continuar discutiendo las implicaciones de la transición que vivimos en términos de pasar de entender las reivindicaciones politicas y sociales de los derechos humanos en tiempos de guerra, a comprenderlos en tiempos de transición a la democracia. Esta comprensión, anuncian, supone pensar en nuevos modelos epistemológicos, y nuevas enunciaciones que permitan comprender qué tipo de herramientas hay ahora para la lucha y qué tipo de proyecto vale la pena construir.

(Diario de campo, Medellín, 14 de Marzo de 2017)

Este coloquio político del IPC reunía, en sus palabras, al Movimiento de Derechos Humanos de Antioquia, pues participaban de él una gran cantidad de movimientos, organizaciones y asociaciones orientadas por la defensa de los Derechos Humanos que asistían a este espacio a dialogar y reflexionar sobre sus propias posicionalidades en este momento de transición. En particular, la conversación tenía que ver con, en un primer momento, enunciar los modos en los que cada organización, haciendo una lectura del contexto, empezaba a redefinir su agenda de trabajo; y en un segundo momento, con pensar en las posibilidades de articulación y solidaridad con las luchas de las otras organizaciones.

Esta conversación estuvo atravesada por los interrogantes a los que hace alusión la viñeta sobre los Derechos Humanos. Aunque históricamente la llegada a Colombia del discurso de los Derechos, acompañado por las plataformas globales para su promoción, sirvió a muchas organizaciones sociales para proteger la vida y la organización de las fuerzas del Estado que pretendieron reprimirlas y desaparecerlas; también es cierto que muchas de estas luchas y reivindicaciones no se agotaban en la promoción del listado de derechos; al contrario, siempre hicieron usos estratégicos de estos discursos globales para ganar espacios políticos locales.

Este momento, y en especial, la conversación sobre la paz, parece ser un momento muy parecido, en donde la coyuntura invita a articular esfuerzos y agendas, pero con unas lecturas muy críticas con respecto a las herramientas que provee el Estado y la comunidad internacional para su realización. Después de todo, son las organizaciones sociales y las comunidades las que están poniendo los muertos de esta paz con el asesinato selectivo y sistemático de líderes-as sociales en todo el territorio ${ }^{6}$. Con el lema Que la paz no nos cueste la vida estas organizaciones

\footnotetext{
${ }^{6}$ A la fecha de escritura de este texto, se contaban 702 líderes-as sociales y 135 excombatientes asesinados desde la firma del acuerdo de paz entre el gobierno colombiano y las FARC-EP
} 
lanzaban interesantes cuestionamientos a las formas y lenguajes en los que debían participar de la coyuntura.

Esta idea de cuestionar las herramientas y estrategias parece tener que ver con la idea propuesta por la lesbiana, feminista y activista negra, Audre Lorde, cuando tituló su conferencia de 1979 con la siguiente expresión: "Las herramientas del amo nunca desarmarán la casa del amo":

Las que estamos fuera del círculo de la definición que esta sociedad da de mujeres aceptables; las que hemos sido forjadas en las encrucijadas de las diferencias -las que somos pobres, que somos lesbianas, que somos negras o que somos más viejas -sabemos que la supervivencia no es una habilidad académica. Es aprender cómo estar en pie sola, impopular y a veces vilipendiada, y cómo hacer causa común con esa otra gente identificada como ajena a las estructuras, con el fin de definir y buscar un mundo en el que todas nosotras podamos prosperar. Es aprender cómo coger nuestras diferencias y convertirlas en potencias. Porque las herramientas del amo no desmantelarán nunca la casa del amo. Nos permitirán ganarle provisionalmente a su propio juego, pero jamás nos permitirán provocar auténtico cambio. Y este hecho sólo resulta amenazador para esas mujeres que todavía definen la casa del amo como su única fuente de apoyo. (LORDE, 1984: 43)

Creo que esta es la apuesta de acción política implícita en este ejercicio de resignificar las herramientas, del discurso de derechos o del discurso de la paz. No se trata solamente del ejercicio epistémico de buscar nuevos horizontes de comprensión de las luchas posibles, o de los

proyectos que vale la pena construir; sino del espacio mismo de conversación sobre las solidaridades que pueden tejerse al margen de estos discursos como estrategia de acción política.

\section{CONCLUSIONES}

A este texto han asistido tanto las voces y relatos encontrados en la exploración del espacio problema configurado por la coyuntura en la educación para la paz, como los discursos dominantes y hegemónicos producidos por la ciencia moderna y reproducidos por las plataformas de gobernanza global y las políticas públicas del Estado colombiano. Esta multiplicidad de lugares desde donde se enuncian significados posibles para esta cartografía no están todos dispuestos de manera uniforme y homogénea en la disputa por el significado de este momento histórico; más bien, lo que he tratado de decir en este texto, y que se manifiesta en la 
multidimensionalidad de este mapa, es que han sido producidos históricamente como ausentes unos lugares de producción de significado sobre la educación para la paz que, de todas formas, persisten hasta hoy como alternativas.

En este punto, y a manera de conclusión, en lo que quiero insistir es en que las experiencias de lucha y solidaridad entre las diferentes comunidades que participan de estos espacios, son en este momento fuente inagotable de ideas y recursos para emprender otros modos de hacer las paces, modos más plurales y más justos con los modos en los que actualmente viven y actúan políticamente poblaciones y territorios explotados por el sistema de género moderno colonial capitalista.

Es justamente en este sentido, y en especial en este momento histórico por el que atraviesa Colombia, que es necesario insistir en unos sentidos posibles de la paz aquí y ahora; es justamente en este momento cuando el relato de la paz se hace frágil y hay tantas luchas en riesgo, que se hace patente la necesidad de volver sobre las alternativas que hoy en los territorios más lejanos, pero también en las calles de las ciudades, se están planteando para que la vida siga siendo posible.

\section{REFERENCÍAS}

APARICIO, Juan Ricardo. 2016. Del archivo y la construcción del territorio: una problematización de los estudios de la memoria. In: SAIZ, Mónika; LOUIS, Tatjana; RINKE, Stefan. Memoria y conflicto. Memorias en conflicto. Intercambios metódicos y teóricos de experiencias locales latinoamericanas. Stuttgart, Verlag Hans-Dieter Heinz, Akamedischer Verlag Stuttgart, Universidad de los Andes.

CASTRO-GÓMEZ, Santiago. 2005. La hybris del punto cero. Bogotá, Editorial Pontificia Universidad Javeriana.

CHAKRABARTI, Dipesh. 2008. La historia subalterna como pensamiento político. In: Mezzadra, Sandro. Estudios postcoloniales. Ensayos fundamentales. Madrid, Traficantes de sueños.

CHAMBERS, Paul. 2013. En busca de las causas del conflicto armado colombiano: analizando los comienzos de una tendencia científico-social. Discusiones Filosóficas, 23: 279-304.

CONGRESO DE COLOMBIA. 2014. Ley n ${ }^{\circ} 1732$ de 2014.

CUNHA, Teresa. 2006. Género paz e interculturalidade - Uma crítica feminista e pós-colonial. Portugal, Acção para a Justiça e Paz (AJPaz).

DEAS, Malcolm. 1999. Intercambios violentos. Reflexiones sobre la violencia política en Colombia. Bogotá, Taurus/Pensamiento. 
ESCOBAR, Arturo. 1996. La invención del tercer mundo: construcción y deconstrucción del desarrollo. Bogotá, Editorial Norma.

FANON, Franz. 1983. Los condenados de la tierra. México, Fondo de cultura económica.

FOUCAULT, Michel. 1988. El sujeto y el poder. Revista mexicana de sociología, México, 50(3): 3-20.

GAlTUNG, Johan. 1996. Peace by Peaceful Means: Peace and Conflict, Development and Civilization. Londres, Sage.

GILLIGAN, Carol. 1982. In a different voice. Massachusetts, Harvard University Press.

GRASA, Rafael. 2010. Cincuenta años de evolución de la investigación para la paz: tendencias y propuestas para observar, investigar y actuar. Barcelona, Generalidad de Catalunya.

GUZMÁN CAMPOS, Germán., et al. 2005. La violencia en Colombia. Bogotá, Taurus.

HARAWAY, Donna. 1995. Ciencia, cyborgs y mujeres. La reinvención de la naturaleza. Madrid, Cátedra.

HARDING, Sandra. 1986/1996. Ciencia y Feminismo. Madrid, Morata.

JIMÉNEZ-OCAMPO, Sandro. 2014. La administración de los efectos de la guerra como tecnología de gobierno: una mirada post-liberal al conflicto armado en Colombia, 1980-2010. Quito, FLACSO.

LANDER, Edgardo. 2000. La colonialidad del saber: eurocentrismo y ciencias sociales. Perspectivas. Buenos Aires, CLACSO.

LORDE, Audre. 1984. Sister Outsider. Nova Iorque, Crossing Press.

LUGONES, María. 2008. Colonialidad y género. Tabula Rasa, (9): 73-101.

MANCHANDA, Rita. 2005. Women's agency in peace building. Economic and political weekly, Mumbai, 40(44/45): 4737-4745.

MENDIA, Irantzu. 2014. La división sexual del trabajo por la paz. Madrid, Editorial Tecnos.

MENESES, María Paula. 2016. Os sentidos da descolonização: uma análise a partir de Moçambique. Opsis, Catalão, 16(1): 26-44.

MIGNOLO, Walter. 2003. Historias locales / diseños globales. Colonialidad, conocimientos subalternos y pensamiento fronterizo. Madrid, Ediciones Akal.

MOHANTY, Chandra. 2008. Bajo los ojos de occidente. In L. S. Hernández, Descolonizando el Feminismo:Teorías y Prácticas desde los Márgenes. Madrid, Cátedra.

; RUSSO, Ann; TORRES, Lourdes. 1991. Third World Women and the Politics of Feminism. Bloomington, Indiana University Press.

MOLANO, Alfredo. 2010. Siguiendo el corte: Relatos de guerras y tierras. Bogotá, Punto de lectura.

OQUIST, Paul. 1978. Violencia, conflicto y política en Colombia. Bogotá, Instituto de Estudios Colombianos.

OYĚWÙMÍ, Oyèrónké. 1997. The Invention of Women: Making an African Sense of Western Gender Discourses. Minneapolis, University of Minnesota Press.

PÉCAUT, Daniel. 2012. Orden y violencia: Colombia 1930-1953. Medellín, Colección Ediciones EAFIT.

PRECIADO, Beatriz. 2008. Cartografías queer: el flâneur perverso, la lesbiana topofóbica y la puta multicartográfica, o como hacer una cartografía 'zorra' con Annie Sprinkle. In: Cortés, José Miguel G. Cartografias Disidentes. Barcelona, SEACEX. 
PUREZA, Jose; CRAVO, Teresa. 2009. Critical Edge and Legitimation in Peace Studies. RCCS Annual Review, Coimbra, (0):1-15.

RICHMOND, Oliver. 2010. Resistance and the post-liberal peace. Millennium: Journal of International Studies, Londres, 38(3): 665-692.

SÁNCHEZ, Gonzalo., et al. 1985. Colombia: violencia y Democracia. Bogotá, Universidad Nacional/Colciencias.

SÁNCHEZ-CORRALES, Natalia et al. 2020. Cartografías de saberes y prácticas de educación para la paz. In: La paz como construcción colectiva. Un recorrido por los aprendizajes y los repertorios de acción. Medellín.

SANTOS, Boaventura de Sousa. 2002. Para uma sociologia das ausencias e uma sociologia das emergências. Revista Critica de Ciencias Sociais, Coimbra, (63): 237-280.

. 2006. Conocer desde el sur. Para una cultura politica emancipatoria. Lima: Fondo Editorial de la Facultad de Ciencias Sociales. . 2010. Descolonizar el saber, reinventar el poder. Montevideo, Ediciones Trilce. . 2011. Epistemologías del sur. Utopía y Praxis Latinoamericana, Maracaibo, (54): 17-39.

SERJE, Margarita. 2011. El revés de la nación: Territorios salvajes, fronteras y tierras de nadie. Bogotá, Ediciones Uniandes.

TICKNER, Arlene. 2012. A Critique of Morgenthau's Principles of Political Realism. In: ART, Robert J.; JERVIS, Robert. International Politics: Enduring Concepts and Contemporary issues, Harlow, Global Edition: 22-34.

TROUILLOT, Michel-Rolph. 1995. Silencing the Past. Power and the Production of History. Boston, Beacon Press. 